\title{
In silico analysis of selected components of grapefruit seed extract against SARS-CoV-2 main protease
}

\author{
Belmina Saric ${ }^{1}$, Nikolina Tomic ${ }^{1}$, Abdurahim Kalajdzic ${ }^{1}$, Naris Pojskic ${ }^{1}$, Lejla Pojskic ${ }^{*}$
}

\begin{abstract}
At the end of December 2019, first identified cases of Severe Acute Respiratory Syndrome Coronavirus 2 (SARS-CoV-2) started emerging. Ever since the emergence of the first case of infection with SARS-CoV-2 or COVID-19, it became the hottest research topic of numerous studies, in which scientists are trying to understand the path of infection, transmission, replication and viral action, all in order of finding a potential cure or vaccine applying various fundamental principles and methodologies. Using in silico method via AutoDock Vina 1.1.2., we analysed the binding affinity of six selected compounds from grapefruit seed extract (GSE) (narirutin, naringin, naringenin, limonin, ascorbic acid and citric acid) to SARS-CoV-2 main protease $\mathrm{M}^{\text {pro }}$ (PDB ID: 6Y84), using acetoside, remdesivir and gallic acid as a positive controls of binding affinity. Results showed highest affinity (rmsd l.b. 0.000; rmsd u.b. 0.000) for narirutin (-10.5), then for naringin (-10.1), acetoside (-10.0), limonin (-9.9), remdesivir (-9.6), naringenin (-8.2), ascorbic acid (-6.7), citric acid (-6.4) and gallic acid (-6.4), all expressed in kcal/mol. Our findings suggest that selected compounds from grapefruit seed extract represent potential inhibitors of SARS-CoV- $2 \mathrm{M}^{\text {pro }}$, but further research is needed as well as preclinical and clinical trials for final confirmation of inhibitory functionality of these compounds.
\end{abstract}

Keywords: Grapefruit seed extract (GSE), SARS-CoV-2, main protease, molecular docking, COVID-19

${ }^{1}$ Institute for Genetic Engineering and Biotechnology, University of Sarajevo, Sarajevo, Bosnia and Herzegovina

*Corresponding Author:

Prof. dr Lejla Pojskic. Institute for Genetic Engineering and Biotechnology, University of Sarajevo, Sarajevo, Bosnia and Herzegovina; E-mail: lejla.pojskic@ingeb.unsa.ba

DOI: $10.2478 /$ ebtj-2021-0015

(๑) 2021 Authors. This work was licensed under the Creative Commons Attribution-NonCommercialNoDerivs 4.0 License.

\section{Introduction}

Since the outbreak of the SARS-CoV-2 virus, from December 2019 until May 2021, more than 157 million people have been infected, and over 3.2 million deaths have been recorded (1). SARS-CoV-2 is a positive-sense single-stranded RNA (+ssRNA) virus which belongs to Betacoronavirus genus of the Coronaviridae family, causing coronavirus disease 2019 (COVID-19) with severe (13.8\%) and critical symptoms (4.7\%) in some cases, including deadly dangerous fatal pneumonia $(2,3)$.

Genomes of some typical Coronaviruses (CoVs), just like SARS-CoV-2 itself, have 6-10 open reading frames (ORFs). The first, the largest open reading frame, encodes 16 non-structural proteins nsps (nsp1-nsp16) (4). Nsp-5 encodes the main protease known as $\mathrm{M}^{\text {pro }}$ (chymotrypsin-like cysteine protease, $3 \mathrm{CL}^{\text {pro }}$ ), with an important role in virus replication and gene expression (5). Chymotrypsin-like cysteine protease (306 amino acid residues) (6) has three domains: catalytic domain, C-terminal domain and N-terminal finger domain, labeled as domain I, domain II and domain III, respectively. Domain III has enzymatic activity and no role in binding (7). The active site of this enzyme contains four sites, S1', S1, S2, and S4 $(8,9)$, while the catalytic site of $\mathrm{M}^{\text {pro }}$ preserves catalytic diad (His41-Cys145) located between domain I and II (7). $\mathrm{M}^{\text {pro }}$ is highly conserved among all of the Coronaviruses (8). Main role of $\mathrm{M}^{\text {pro }}$ in viral replication is its proteolytic activity. Proteolytic cleavage is performed by papain-pike protease $\left(\mathrm{PL}^{\mathrm{pro}}\right)$ and the main protease $\left(\mathrm{M}^{\mathrm{pro}}\right)$, releasing functional polypeptides from the ppla and pplab polyproteins $(10,11)$. 
Because of its important role in viral replication and its highly conserved catalytic domains, main protease is a potential drug target (11).

Finding an effective cure or vaccine against SARS-CoV-2 represents a great challenge to humanity. In order to stop the global pandemic, numerous studies and trials of potential drugs against SARS-COV-2 are underway (12).

One such potential antiviral substance is grapefruit seed extract (GSE), a commercial product made from grapefruit seeds and pulp, that is often used as a dietary supplement (13). Grapefruit (Citrus $\times$ paradisi, Macfad) is a subtropical fruit that belongs to the Rutaceae family. It has originated as a natural hybrid of Citrus maxima, Merr. and Citrus $\times$ sinensis, (L.) Osbec from Barbados (14). GSE has strong antibacterial, antifungal, antiviral, liver-protective, antioxidant, neuroprotective, cardioprotective activity and many other bioactive properties, because of its high content of flavonoids, ascorbic acid, tocopherol, citric acid, limonoids, sterols, minerals and numerous other bioactive substances (13). The secondary metabolites of grapefruit seeds are predominately limonoids and flavonoids, such as limonin, naringin, narirutin or naringenin and hesperidin $(14,15,16)$. According to Komura et al. (2019) GSE showed anti-viral activity against enveloped viruses, but not against non-enveloped viruses (17). The research by Go et al. (2020) showed that grapefruit seed extract (GSE) plus xylitol as a nasal spray solution can be used as a potential adjunct treatment of COVID-19 (18).

In silico analysis is a promising way of detecting potential drugs, virtually allowing us to understand the receptor-ligand interaction (19). Currently, a large number of in silico studies are underway. All of them have a common purpose, to find potential inhibitor of drug targets of SARS-CoV-2 virus. Diverse class of natural compounds such as terpenoids, coumarins, flavonoids, glycosides, phenols and polyphenols, catechins, etc. are involved in those studies as a potential inhibitors of SARSCoV-2 M $M^{\text {pro }}(20,21,22)$. Besides the natural compounds, many synthetic compounds are also included by in silico analysis of drug repurposing as a potential inhibitors. $(23,24)$. According to Teli et al. (2021) nine natural compounds with the best docking scores are selected as a potential inhibitors of SARSCoV-2 main protease $\mathrm{M}^{\text {pro }}$. Those components are procyanidin A3, rutin, solanine, procyanidin A4, procyanidin B4, hypericin, quercetagetin, procyanidin, and astragalin, that belong to different groups of bioactive substances such as bio-flavonoids, flavones, quinone, etc. They found out that acetoside and curcumin inhibit $\mathrm{M}^{\text {pro }}$ covalently (20). In certain previous studies, ritonavir and remdesivir demonstrated the best docking scores, which is quite interesting, due to their use as a potential therapeutic agents in preclinical and clinical trials for treatment of COVID-19 patients (23). Also, ritonavir-lopinavir, a clinically approved drug that came from new clinical trial as a drug for COVID-19, in the study of Kumar et al. (2020) showed best binding affinity $(-10.6 \mathrm{kcal} / \mathrm{mol})$. Except for ritonavir-lopinavir, tipranavir also showed the second best binding affinity (-8.7) (24). One molecular docking analysis suggested com- pounds such as: naringin (flavanones), quercetin (flavonols), capsaicin, psychotrine and gallic acid as potential inhibitory compounds against $\mathrm{M}^{\text {pro }}$ of SARS-CoV-2 based on their binding energy scores. Naringin and naringenin belong to flavonoids, the secondary metabolites which have strong anti-in flammatory and antioxidant activities and are also found in grapefruit seed extract (22). Those are just some of the many molecular docking studies that are used for drug repurposing and finding potential adjuvant in treatment of COVID-19.

The aim of this study was to analyze potential inhibitory effect of selected natural compounds from grapefruit seed extract (GSE) using SARS-CoV-2 main protease $\left(\mathrm{M}^{\text {pro }}\right)$ as a target protein.

\section{Materials and Methods}

In this study, the virtual interaction of the SARS-CoV-2 main protease $\left(\mathrm{M}^{\text {pro }}\right)$ and 6 selected components of grapefruit seed extract (naringin, narirutin, naringenin, limonin, ascorbic acid and citric acid) was examined. As positive controls of binding affinity, we used acetoside, remdesivir and gallic acid, because of their high binding affinity against $\mathrm{M}^{\text {pro }}$ in other molecular docking studies $(20,21,23)$. Also, acetoside covalently inhibited $\mathrm{M}^{\text {pro }}$ in study of Teli et al. (2021), while remdesivir is used in many clinical trials for treating COVID-19 patients $(25,26)$.

The three-dimensional crystal structure of SARS-CoV-2 main protease Mpro (PDB ID: 6Y84) was retrieved from Protein Data Bank in PDB format (27). The three dimensional structures of selected components of grapefruit seed extract (ligands) were retrieved from the PubChem (28) chemical database as SDF format and then converted to PDB three-dimensional format using online software Online SMILES Translator and Structure File Generator (29). PubChem CID of naringin (442428), naringenin (932), narirutin (442431), limonin (179651), ascorbic acid (54670067), citric acid (311), acetoside (24978601), remdesivir (121304016) and gallic acid (370), were used.

Preparation of target protein $\mathrm{M}^{\text {pro }}$ and ligands, was performed using the software AutoDock Tools, precisely MGLTools version 1.5.6. (30), wherein the molecules were converted to pdbqt format. Using Auto Dock Tools we removed all water molecules from protein and added polar hydrogen atoms as well as Kollmans charges.

Molecular docking was performed using AutoDock Vina 1.1.2. following standard procedures (31). We selected eight amino acid residues within chain $\mathrm{A}$ of the main protease (Mpro) as the catalytic binding site, namely His41, Phe140, Gly143, Ser144, Cys145, His163, His164 and Glu166, following the relevant literature $(10,12)$. Cys145 - His41 dyad is a highly conserved region that forms an active site that has a catalytic function (7). Cys145, Gly143 and Ser144 are S1 residues which makes the oxyanion hole. His163 is yet another S1 residue, while Glu166 is a part of S2. According to Kumar et al. (2020) SARS-CoV-2 $\mathrm{M}^{\text {pro }}$ has strong bond interaction with ligand (improved-a-ketoamide) due to formed hydrogen bond between the His41 active site and residues such as His164, Glu166, 
Gly143, His163, Cys145, His41, and Phe140 (10, 12). Grid box (40 $\AA$ x $40 \AA$ x $40 \AA$ ) (32) was set to cover the selected amino acid residues, with $\mathrm{x}, \mathrm{y}$ and $\mathrm{z}$ coordinates as $11.500,-2.000$ and 15.000 respectively. Dial spacing (angstrom - $\AA$ ), in each analysis, was set to 0.517 centering around hotspot residues. A program called PyMOL 2.4. was used to review the final results (33). The docking was performed using an exhaustiveness value of 8 (34). After preparation of input molecules, grid box and configuration file, Vina program was run through Command Prompt.

\section{Results}

AutoDock Vina produced nine binding modes for every tested ligand with particular docking score (binding energy). The most stable binding mode for ligand is the mode with the least binding energy. The best binding modes and corresponding affinities of selected compounds of grapefruit seed extract (narirutin, naringin, naringenin, limonin, ascorbic acid abd citric acid) and binding affinities of positive controls (acetoside, remdesivir and gallic acid) against main protease of SARS-CoV-2 are presented in Table 1. These positive controls of binding affinities were chosen because of their high binding affinity against $\mathrm{M}^{\text {pro }}$ in other molecular docking studies.

The highest binding affinity was obtained for flavonoid nariru-
(Figure 1B; Figure 2B; Figure 3B).

Also, the naringin and naringenin showed similarity for binding modes to main protease close to position of Ser284 (Fig 2B; Fig 4B), just like ascorbic acid and citric acid for binding modes close to position of Glu290 and Glu288 (Figures: 5B, 6B).

Except for these similar interactions, narirutin showed interaction close to position Gly138 and naringenin close to position Arg4 (Fig 1B; Fig 4B).

Visualisation of the SARS-CoV-2 main protease with positive controls (acetoside, remdesivir and gallic acid) via PyMOL 2.4. showed that all three had interactions close to positions of Lys137 and Gln127 (Fig 7B; Fig 8B; Fig 9B) and also limonin interacted near position Lys137 (Fig 3B).

\section{Discussion}

Numerous studies and clinical trials about vaccines as well as a potential antiviral drugs are underway to hopefully put an end to the SARS-CoV-2 pandemic $(35,36)$. In silico prediction of receptor-ligand interaction and their affinity of binding has become an excellent way to examine new potential drugs against SARS-CoV-2 leading to the improvement of the patient's condition as well as reducing the number of deaths (37).

There are five approaches of viral inhibition at different lev-

Table 1. Best docking scores (lowest binding energy) of selected compounds of grapefruit seed extract (GSE) as well as of the positive controls and their distance from best mode

\begin{tabular}{|c|c|c|c|}
\hline \multirow[b]{2}{*}{ Ligands } & \multirow[b]{2}{*}{ Affinity (kcal/mols) } & \multirow[b]{2}{*}{ rmsd l.b. ${ }^{\dagger}$} & \multirow[b]{2}{*}{ rmsd u.b } \\
\hline & & & \\
\hline Narirutin & -10.5 & 0.000 & 0.000 \\
\hline Naringin & -10.1 & 0.000 & 0.000 \\
\hline Naringenin & -8.2 & 0.000 & 0.000 \\
\hline Limonin & -9.9 & 0.000 & 0.000 \\
\hline Ascorbic acid & -6.7 & 0.000 & 0.000 \\
\hline Citric acid & -6.4 & 0.000 & 0.000 \\
\hline Acetoside & -10.0 & 0.000 & 0.000 \\
\hline Remdesivir & -9.6 & 0.000 & 0.000 \\
\hline Gallic acid & -6.4 & 0.000 & 0.000 \\
\hline
\end{tabular}

Legend: $\uparrow$ root mean square deviation lower bound; $\ddagger$ root mean square deviation upper bound; $\$$ - kilocalorie per mole;

- compounds of GSE; • positive controls

tin (-10.5, rmsd 1.b. 0.000; rmsd u.b. 0.000) followed by a very similar affinity of naringin (-10.1, rmsd l.b. 0.000 ; rmsd u.b. 0.000) and slightly lower of limonin (-9.9, rmsd l.b. 0.000; rmsd u.b. 0.000). These binding affinities were similar to values of binding affinites for positive controls acetoside $(-10.0, \mathrm{rmsd} 1 . \mathrm{b}$. 0.000; rmsd u.b. 0.000) and remdesivir (-9.6, rmsd 1.b. 0.000; rmsd u.b. 0.000). The binding affinities acquired for citric acid (-6.4, rmsd l.b. 0.000; rmsd u.b. 0.000) and positive control gallic acid (-6.4, rmsd l.b. 0.000; rmsd u.b. 0.000).

Visualized interactions showed similarity in narirutin, naringin, limonin binding modes to $\mathrm{M}^{\text {pro }}$ close to position of Lys5 els: 1.virus binding to the host receptor; 2 . viral endocytosis; 3 . neutralizing the virus particle; 4 . targeting the viral structural proteins like the membrane, envelope and nucleocapsid protein and 5. restoration of host's innate immunity (38).

Some of the potential drug targets of SARS-CoV-2 are spike protein (S), small envelope protein (E), matrix protein (M) and nucleocapsid protein $(\mathrm{N})$. They all have a role in virus survival and propagation, and that is why their inhibition can lead to virus decay, which makes them ideal for drug targeting $(35,37)$. However, the best key targets in developing the antiviral drugs are the two proteases, $3 \mathrm{CL}^{\text {pro }}$ (chymotrypsin-like cysteine pro- 


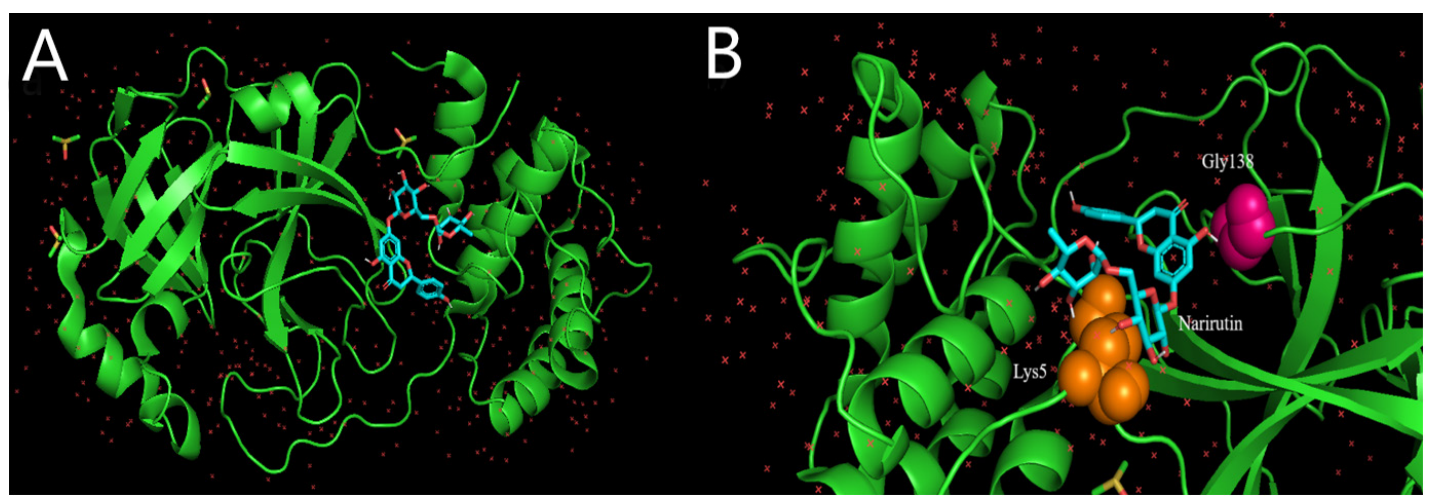

Figure 1. A-B. Visualisation of the SARS-CoV-2 main protease (A) (PDB ID: 6Y84) with ligand narirutin and their best mode binding interactions (B) via PyMOL 2.4.

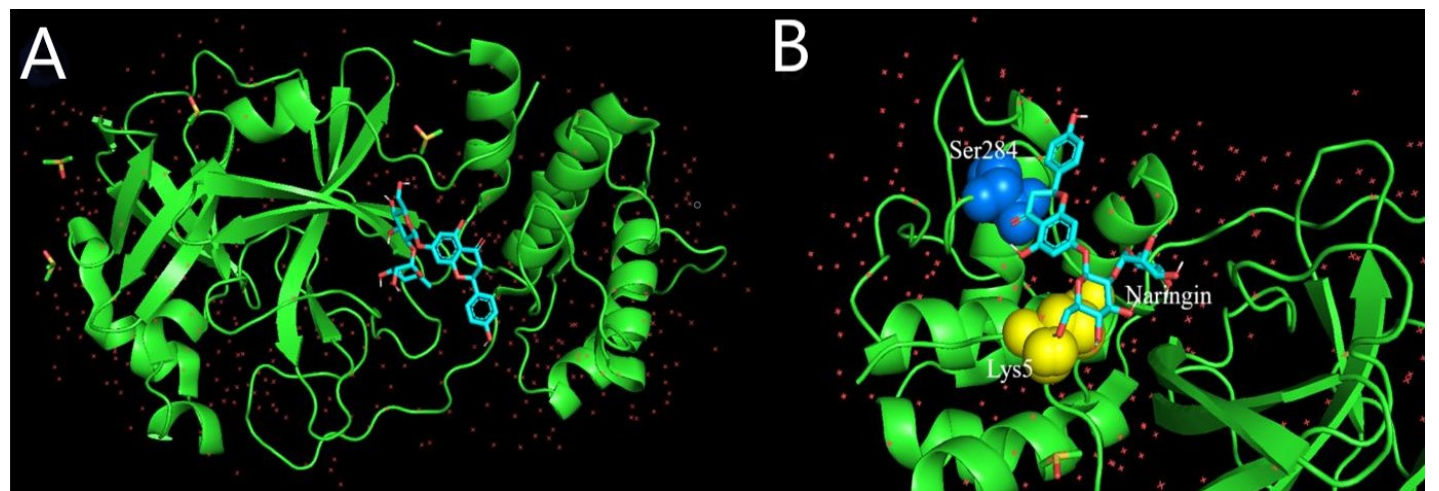

Figure 2. A-B. Visualisation of the SARS-CoV-2 main protease (A) (PDB ID: 6Y84) with ligand naringin and their best mode binding interactions (B) via PyMOL 2.4.

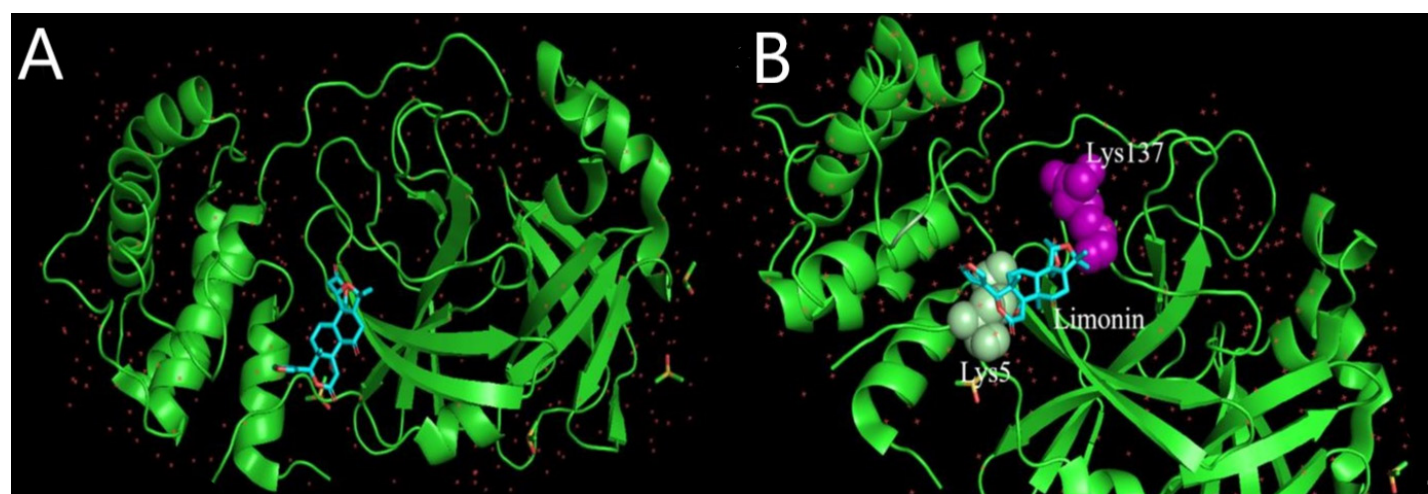

Figure 3. A-B. Visualisation of the SARS-CoV-2 main protease (A) (PDB ID:6Y84) with ligand limonin and their best mode binding interactions (B) via PyMOL 2.4.
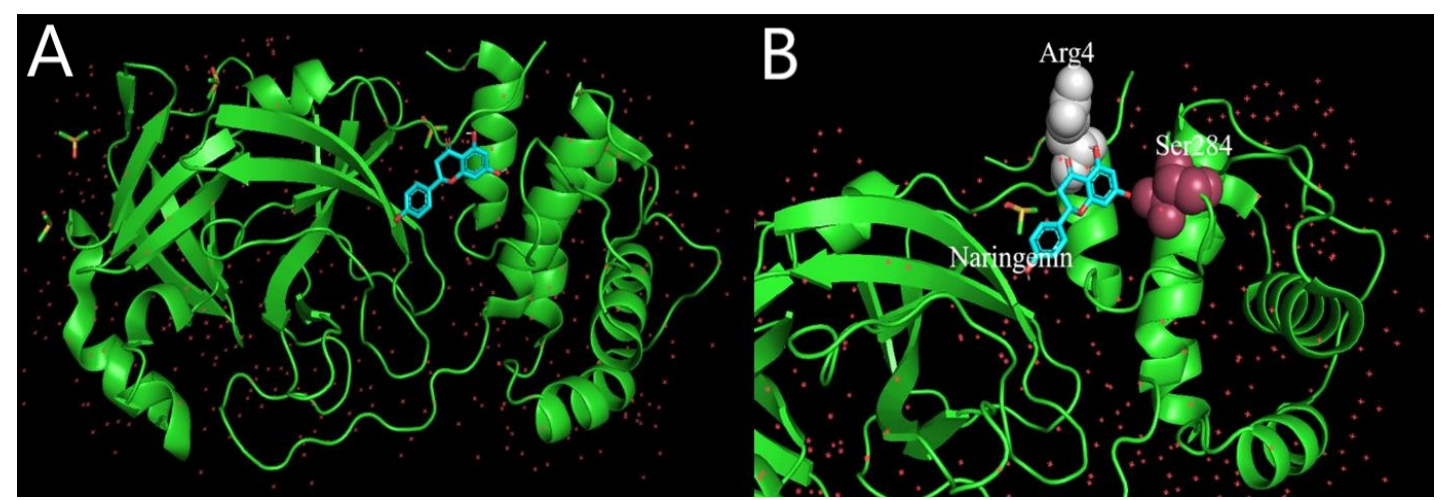

Figure 4. A-B. Visualisation of the SARS-CoV-2 main protease (a) (PDB ID:6Y84) with ligand naringenin and their best mode binding interactions (b) via PyMOL 2.4 


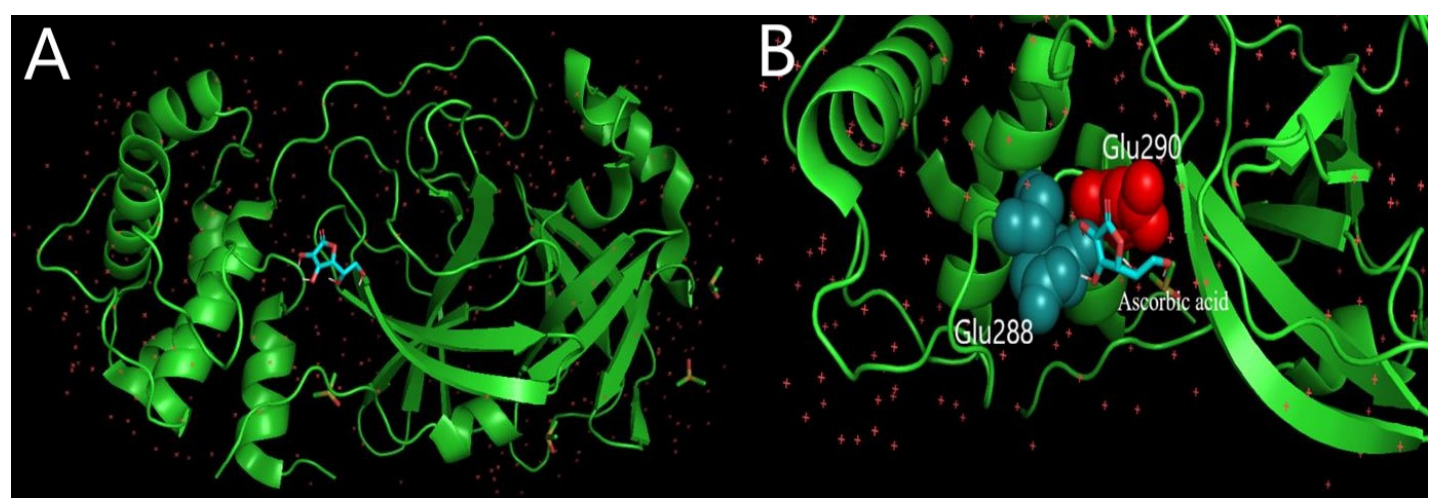

Figure 5. A-B. Visualisation of the SARS-CoV-2 main protease (a) (PDB ID:6Y84) with ligand ascorbic acid and their best mode binding interactions (b) via PyMOL 2.4.

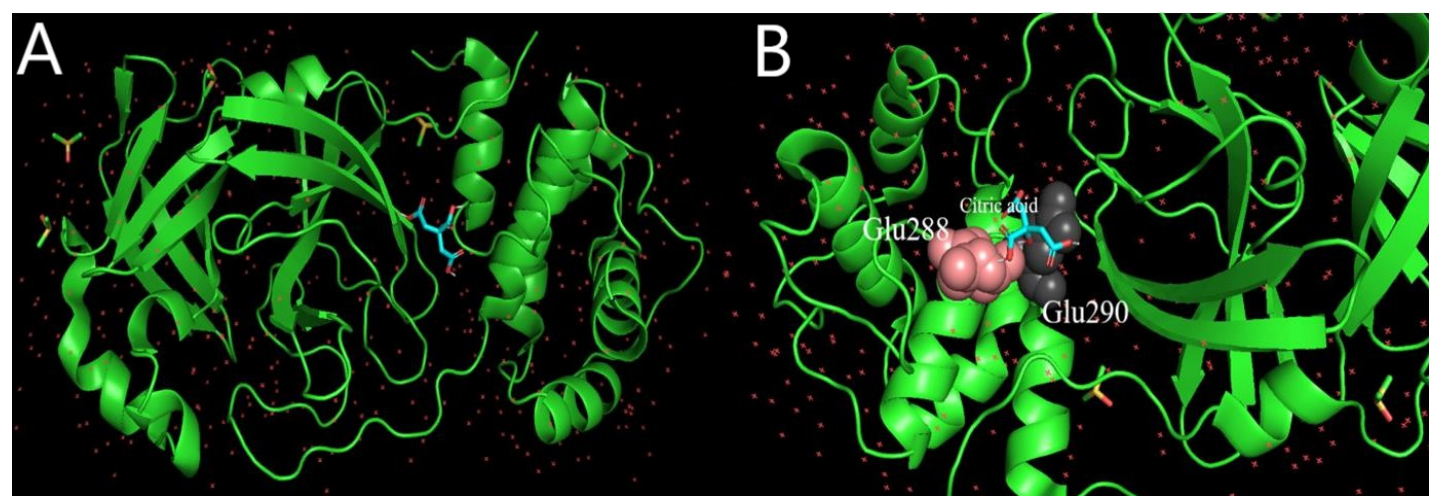

Figure 6. A-B. Visualisation of the SARS-CoV-2 main protease (a) (PDB ID:6Y84) with ligand citric acid and their best mode binding interactions (b) via PyMOL 2.4.

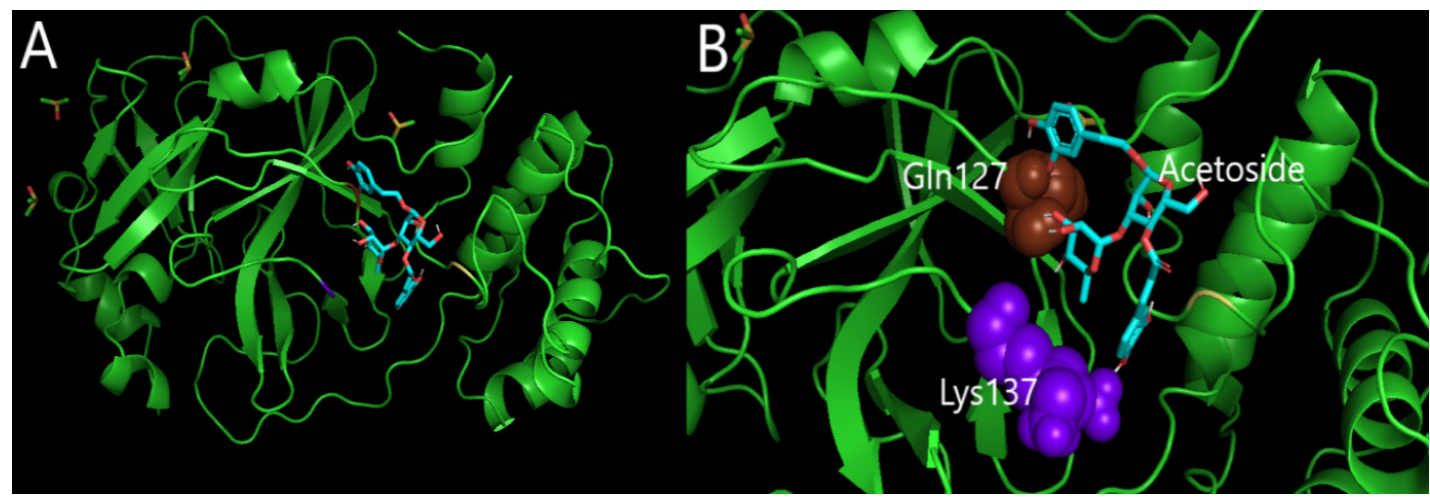

Figure 7. A-B. Visualisation of the SARS-CoV-2 main protease (a) (PDB ID:6Y84) with ligand acetoside and their best mode binding interactions (b) via PyMOL 2.4.

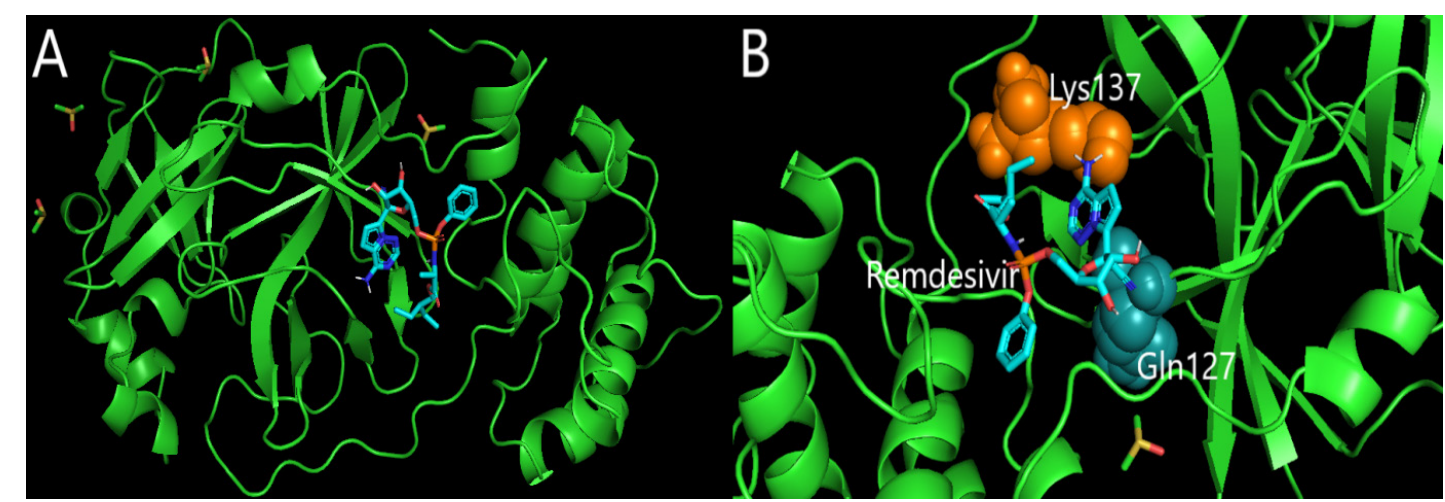

Figure 8. A-B. Visualisation of the SARS-CoV-2 main protease (a) (PDB ID:6Y84) with ligand remdesivir and their best mode binding interactions (b) via PyMOL 2.4. 


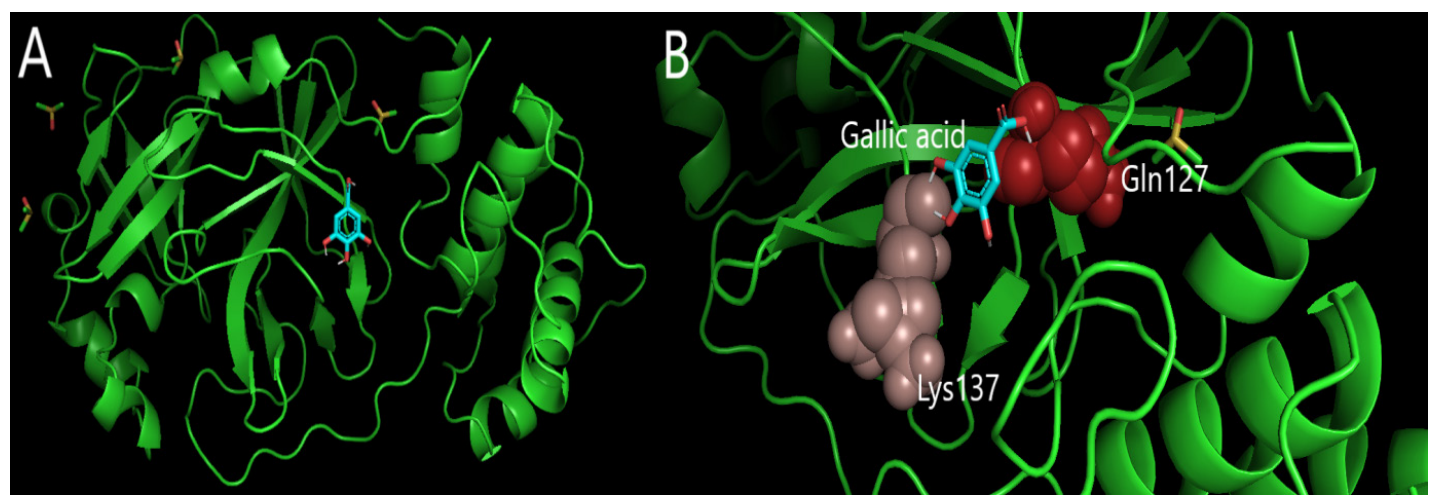

Figure 9. A-B. Visualisation of the SARS-CoV-2 main protease (a) (PDB ID:6Y84) with ligand gallic acid and their best mode binding interactions (b) via PyMOL 2.4.

tease, also known as $\mathrm{M}^{\text {pro }}$ ) and PL ${ }^{\text {pro }}$ (papain-like protease) (39). These proteases have a critical role in viral replication, whereby their proteolytic activities lead to the formation of functional non-structural proteins (11).

In this study, we analyzed virtual interaction between selected compounds from grapefruit seed extract (narirutin, naringin, naringenin, limonin, ascorbic acid, citric acid) as a potential inhibitors against main protease $\mathrm{M}^{\text {pro }}$ of SARS-CoV-2 using molecular docking method. As positive controls we used acetoside, remdesivir and gallic acid, because of their high binding affinity in other molecular docking studies and potential inhibitory effect against $\mathrm{M}^{\text {pro }}(20,22,23)$. The binding affinity of selected compounds ranged, from the highest for narirutin $(-10.5 \mathrm{kcal} / \mathrm{mol})$ to the lowest for citric acid $(-6.4 \mathrm{kcal} /$ $\mathrm{mol})$. The binding affinities for narirutin $(-10.5 \mathrm{kcal} / \mathrm{mol})$ and naringin $(-10.1 \mathrm{kcal} / \mathrm{mol})$ were slightly higher than binding affinity of positive control acetoside $(-10.0 \mathrm{kcal} / \mathrm{mol})$. According to Teli et al. (2021) acetoside showed the second hightest docking score against $\mathrm{M}^{\text {pro }}(-11.974 \mathrm{kcal} / \mathrm{mol})$ and also they found out that acetoside covalently inhibited $\mathrm{M}^{\text {pro }}$ of SARS-CoV-2 virus (20). The value of limonin binding affinity was not negligible either $(-9.9 \mathrm{kcal} / \mathrm{mol})$ as well as the binding affinity of positive control remdesivir $(-9.6 \mathrm{kcal} / \mathrm{mol})$, while in the study of Ibrahim et al. (2021) it showed lower binding affinity (-8.2 $\mathrm{kcal} / \mathrm{mol}$ ), but it was the second highest value of binding affinity in their analysis (23). Remdesivir is promising potential drug which was used in many clinical trials for treating COVID-19 patients $(25,26)$. It is very interesting that narirutin, naringin, and limonin after visualization via PyMOL 2.4. had similar binding interaction of $\mathrm{M}^{\text {pro }}$ close to position of Lys5. Study by Vijayakumar et al. (2020) showed that residue Lys5 is one of the regulatory sites for interaction (40). Visualised interaction between $\mathrm{M}^{\text {pro }}$ and the positive controls we used in this study (acetoside, remdesivir, gallic acid), indicated the same positions that included Lys137 and Gln127. Also, limonin showed interactions near position Lys137. Binding affinity of positive control gallic acid $(-6.4 \mathrm{kcal} / \mathrm{mol})$ was the lowest, similar to binding affinites of citric acid $(-6.4 \mathrm{kcal} / \mathrm{mol})$ and ascorbic acid $(-6.7 \mathrm{kcal} / \mathrm{mol})$, despite gallic acid having the highest docking score $(-17.45 \mathrm{kcal} / \mathrm{mol})$ in the study of Alrasheid et al. (2021). Also, in that study, naringin showed binding affinity of -14.5 $\mathrm{kcal} / \mathrm{mol}$ (22). Previous research that included in silico analysis with the main protease of SARS-CoV-2 showed a binding affinity for naringin of $-8.1 \mathrm{kcal} / \mathrm{mol}$ just like for narirutin, and for naringenin binding affinity was $-8.3 \mathrm{kcal} / \mathrm{mol}$ (41). According to Vardhan, Sahoo (2020) results of molecular docking for binding affinty of limonin was $-8.7 \mathrm{kcal} / \mathrm{mol}$ (42).

Some of the studies observed the antiviral effect of grapefruit seed extract (17). The research by Go et al. (2020) showed that grapefruit seed extract (GSE) plus xylitol as a nasal spray solution can be used as a potential adjunct treatment of COVID-19. In their study, the three patients with high risk of morbidity and mortality, after intranasal usage of GSE plus xylitol, after a few days had an improvement in their symptoms and a reduced number of days required for elimination of viral particles on nasopharyngeal epithelium. Additional studies are needed to evaluate the efficacy of suchlike applications and antiviral delivery in prevention of SARS-CoV-2 attachment and progression of infection (18). Our findings on antiviral activity of grapefruit extract, both fractional and combined suggest it could be used as a highly biologically potent adjuvant treatment for COVID-19, after further investigation of the mechanisms of action as well as the potential side effects. Notably, the assessment of synthetic derivatives of citrus extracts suggest that natural extracts as compounds, have proven to be more effective as treatment (antibacterial or cytotoxic) than synthetically obtained pure derivatives or isolated components (43).

\section{References}

1. WHO Coronavirus Disease (COVID-19) Dashboard. [Cited 2021 May 09]. Available from: https://covid19.who. int/.

2. Ouassou H, Kharchoufa L, Bouhrim M, Daoudi NE, Imtara H, Bencheikh N, et al. The Pathogenesis of Coronavirus Disease 2019 (COVID-19): Evaluation and Prevention. J Immunol Res. 2020;2020:7.

3. The Novel Coronavirus Pneumonia Emergency Response Epidemiology Team The Epidemiological Characteristics of an Outbreak of 2019 Novel Coronavirus Diseases (COVID-19)-China. China CDC Weekly. 2020;2:113-122.

4. Woo PCY, Huang Y, Lau SKP, Yuen K-Y. Coronavirus Genomics and Bioinformatics Analysis. Viruses. 
2010;2(8):1804-1820.

5. Xue X, Yu H, Yang H, Xue F, Wu Z, Shen W, et al. Structures of Two Coronavirus Main Proteases: Implications for Substrate Binding and Antiviral Drug Design. J Virol. 2007;82(5), 2515-2527.

6. Motiwale M, Yadav NS, Kumar S, Kushwaha T, Choudhir G, Sharma S, et al. Finding potent inhibitors for COVID-19 main protease (Mpro): an in silico approach using SARS-CoV-3CL protease inhibitors for combating CORONA. J Biomol Struct Dyn. 2020;1-12.

7. Zhang L, Lin D, Sun X, Curth U, Drosten C, Sauerhering $\mathrm{L}$, et al. Crystal structure of SARS-CoV-2 main protease provides a basis for design of improved a-ketoamide inhibitors. Science. 2020; 368(6489):409-412.

8. Yang H, Xie W, Xue X, Yang K, Ma J, Liang W, et al. Correction: Design of Wide-Spectrum Inhibitors Targeting Coronavirus Main Proteases. PLoS Biol. 2005;3(11):e428.

9. Sacco MD, Ma C, Lagarias P, Gao A, Townsend JA, Meng X, et al. Structure and inhibition of the SARSCoV-2 main protease reveal strategy for developing dual inhibitors against Mpro and cathepsin L. Sci Adv. 2020;6(50):eabe0751.

10. Ul Qamar M, Alqahtani S, Alamri MA, Chen L. Structural basis of SARS-CoV-2 3CLpro and anti-COVID-19 drug discovery from medicinal plants. J Pharm Anal. 2020;10(4):313-319.

11. Anand K, Ziebuhr J, Wadhwani P, Mesters JR, Hilgenfeld R. Coronavirus Main Proteinase (3CLpro) Structure: Basis for Design of Anti-SARS Drugs. Science. 2003;300(5626):1763-1767.

12. Kumar Y, Singh H, Patel CN. In silico prediction of potential inhibitors for the main protease of SARS-CoV-2 using molecular docking and dynamics simulation based drug-repurposing. J Infect Public Health. 2020;13(9):12101223.

13. Cvetnic Z, Vladimir-Knezevic S. Antimicrobial activity of grapefruit seed and pulp ethanolic extract. Acta Pharm. 2004;54:243-250.

14. Shahnawaz A, Rattanpal HS, Singh G. Diversity assessment of grapefruit (citrus $\times$ paradisi) and tangelo (citrus $\times$ tangelo) under indian conditions using physico-chemical parameters and ssr markers. App Ecol Environ Res. 2018;16(5):5343-5358.

15. Notice to the US Food and Drug Administration that the use of Vancitrix ${ }^{\mathrm{TN}}$, a glycerin Citrus Extract, is Generally Recognized as Safe. [Cited 2021 Feb 27]. Available from: https://www.fda.gov/media/99981/download.

16. Cardellina JH. Grapefruit Seed Extract Laboratory Guidance Document. American Botanical Council. 2017. [Cited 2021 Feb 27]. Available from: http://cms.herbalgram. org/BAP/LGD/ABC-LGDs-GFSE-CC-05092017-v5.pdf

17. Komura M, Suzuki M, Sangsriratanakul N, Ito M, Takahashi S, Alam MS, et al. Inhibitory effect of grapefruit seed extract (GSE) on avian pathogens. J Vet Med Sci. 2019;81(3):466-472.
18. Go CC, Pandav K, Sanchez-Gonzalez MA, Ferrer G. Potential Role of Xylitol Plus Grapefruit Seed Extract Nasal Spray Solution in COVID-19: Case Series. Cureus. 2020;12(11):e11315.

19. Shalayel M, Al-Mazaideh G, Aladaileh SH, Al-Swailmi F, Al-Thiabat M. Vitamin D is a potential inhibitor of COVID-19: In silico molecular docking to the binding site of SARS-CoV-2 endoribonuclease Nsp15. Pak J Pharm Sci. 2020;33(5):2179-2186.

20. Teli DM, Shah MB, Chhabria MT. In silico Screening of Natural Compounds as Potential Inhibitors of SARS-CoV-2 Main Protease and Spike RBD: Targets for COVID-19. Front Mol Biosci. 2021;7:429.

21. Sharma S, Deep S. In-Silico Drug Repurposing for Targeting SARS-CoV-2 Mpro. ChemRxiv. 2020; Preprint. Available from: https://doi.org/10.26434/chemrxiv.12210845.v1

22. Alrasheid AA, Babiker MY, Awad TA. Evaluation of certain medicinal plants compounds as new potential inhibitors of novel corona virus (COVID-19) using molecular docking analysis. In Silico Pharmacol. 2021; 9(1):10.

23. Ibrahim MAA, Abdelrahman AHM, Allemailem KS, Almatroudi A, Moustafa MF, Hegazy MEF. In Silico Evaluation of Prospective Anti-COVID-19 Drug Candidates as Potential SARS-CoV-2 Main Protease Inhibitors. Protein J. 2021.

24. Kumar Y, Singh H, Patel CN. In silico prediction of potential inhibitors for the main protease of SARS-CoV-2 using molecular docking and dynamics simulation based drug-repurposing. J Infect Public Health. 2020;13(9).

25. Garibaldi BT, Wang K, Robinson ML, et al. Comparison of Time to Clinical Improvement With vs Without Remdesivir Treatment in Hospitalized Patients With COVID-19. JAMA Netw Open. 2021;4(3):e213071.

26. Lai C-C, Chen C-H, Wang C-Y, Chen, Ya-Hui Wang K-H, Hsueh P-R. Clinical efficacy and safety of remdesivir in patients with COVID-19: a systematic review and network meta-analysis of randomized controlled trials. J Antimicrob Chemother. 2021; dkab093.

27. RCSB Protein Data Bank. [Cited 2021 Feb 27]. Available from: https://www.rcsb.org/.

28. PubChem. [Cited 2021 Feb 27]. Available from https:// pubchem.ncbi.nlm.nih.gov/.

29. Online SMILES Translator and Structure File Generator. [Cited 2021 Feb 27]. Available from: https://cactus.nci.nih. gov/translate/.

30. MGLTools. [Cited 2021 Feb 27]. Available from http:// mgltools.scripps.edu/downloads.

31. Trott O, Olson, A. AutoDock Vina: improving the speed and accuracy of docking with a new scoring function, efficient optimization and multithreading. J Comput Chem. 2010;31(2):455-461.

32. Narkhede RR, Pise AV, Cheke RS, Shinde SD. Recognition of Natural Products as Potential Inhibitors of COVID-19 Main Protease (Mpro): In-Silico Evidences. Nat Prod Bioprospect. 2020a;10:297-306. 
33. The PyMOL Molecular Graphics System, Version 2.0 Schrödinger, LLC. [Cited 2021 Feb 27]. Available from https://pymol.org/2/.

34. Narkhede RR, Cheke RS, Ambhore JP, Shinde SD. The Molecular Docking Study of Potential Drug Candidates Showing Anti-COVID-19 Activity by Exploring of Therapeutic Targets of SARS-CoV-2. EJMO. 2020b;4(3):185-195.

35. Dong Y, Dai T, Wei Y, Zhang L, Zheng M, Zhou F. A systematic review of SARS-CoV-2 vaccine candidates. Signal Transduct Targeted Ther. 2020;5:237.

36. Iacob S, Iacob DG. SARS-CoV-2 Treatment Approaches: Numerous Options, No Certainty for a Versatile Virus. Front Pharmacol. 2020;11:1224.

37. Afriza D, Suriyah WH, Ichwan SJA. In silico analysis of molecular interactions between the antiapoptotic protein survivin and dentatin, nordentatin, andquercetin. J Phys Conf Ser. 2018;1073(3):032001.

38. Saxena A. Drug targets for COVID-19 therapeutics: Ongoing global efforts. J Biosci. 2020;45(1):87.

39. Gil C, Ginex T, Maestro I, Nozal V, Barrado-Gil L, Cuesta-Geijo MA, et al. COVID-19: Drug Targets and Potential Treatments. J Med Chem. 2020;63(21):12359-12386.

40. Vijayakumar BG, Ramesh D, Joji A, Prakasan JJ, Kannan T. In silico pharmacokinetic and molecular docking studies of natural flavonoids and synthetic indole chalcones against essential proteins of SARS-CoV-2. Eur J Pharmacol. 2020;886:173448.

41. Cherrak SA, Merzouk H, Mokhtari-Soulimane N. Potential bioactive glycosylated flavonoids as SARS-CoV-2 main protease inhibitors: A molecular docking and simulation studies. PLOS ONE. 2020;15(10):e0240653.

42. Vardhan S, Sahoo SK. In silico ADMET and molecular docking study on searching potential inhibitors from limonoids and triterpenoids for COVID-19. Comput Biol Med. 2020;124:103936.

43. Gualdani R, Cavalluzzi MM, Lentini G, Habtemariam S. The Chemistry and Pharmacology of Citrus Limonoids. Molecules. 2016;21(11):1530. 